\title{
Detection rates of precancerous and cancerous cervical lesions within one screening round of primary human papillomavirus DNA testing: prospective randomised trial in Finland
}

\author{
(c) $\underset{1}{(1)(8)}$ OPEN ACCESS
}

Maarit K Leinonen scientist ${ }^{1}$, Pekka Nieminen chief physician ${ }^{2}$, Stefan Lönnberg scientist ${ }^{1}$, Nea Malila director ${ }^{13}$, Matti Hakama research professor ${ }^{13}$, Arun Pokhrel scientist ${ }^{4}$, Pekka Laurila chief physician $^{5}$, Jussi Tarkkanen physician ${ }^{5}$, Ahti Anttila research director ${ }^{1}$

${ }^{1}$ Mass Screening Registry, Finnish Cancer Registry, FI-00130 Helsinki, Finland; ${ }^{2}$ Department of Obstetrics and Gynecology, Helsinki University Central Hospital, Espoo, Finland; ${ }^{3}$ Tampere School of Health Sciences, University of Tampere, Tampere, Finland; ${ }^{4}$ Finnish Cancer Registry;

${ }^{5}$ Department of Pathology, HUSLAB, Helsinki University Central Hospital, Helsinki

\begin{abstract}
Objective To compare the detection rates of precancerous and cancerous cervical lesions by human papillomavirus (HPV) DNA testing and by conventional cytology screening.

Design Prospective randomised trial. Two cohorts were followed over one screening round of five years, screened initially by primary HPV DNA testing or by primary Pap test.
\end{abstract}

Setting Population based programme for cervical cancer screening in Finland.

Participants Women aged 25-65 years invited for screening in 2003-07 (101 678 in HPV arm; 101747 in conventional cytology arm).

Intervention Women were randomly allocated (1:1) to primary HPV DNA screening followed by cytology triage if they had positive results, or to primary cytology screening. Screening method was disclosed at the screening visit. Trial personnel involved were aware of all test results.

Main outcome measures Cumulative detection rates of cervical intraepithelial neoplasia (CIN), adenocarcinoma in situ (AIS), and invasive cervical cancer before the second screening (after five years) or before 31 December 2008. Lesions detected at screening and during the five year interval were included.

Results 1010 and 701 precancerous or cancerous lesions were detected during an average follow-up of 3.6 years in the HPV and cytology arms, respectively. Among invited women, the hazard ratio was 1.53 (95\% confidence interval I.28 to 1.84) for CIN grade 1, 1.54 (1.33 to 1.78) for CIN 2, 1.32 (1.09 to 1.59) for CIN 3 or AIS, and 0.81 (0.48 to 1.37) for cervical cancer. In 25-34 year old participants, the cumulative hazard (or cumulative detection rate) was 0.0057 (0.0045 to 0.0072) for HPV screening versus $0.0046(0.0035$ to 0.0059$)$ for conventional screening; corresponding data for women aged 35 years and older were 0.0022 (0.0019 to 0.0026$)$ and 0.0017 (0.0014 to 0.0021$)$, respectively.

Conclusions Primary HPV DNA screening detects more cervical lesions than primary cytology within one screening round of five years. Even if the detection rate of CIN 3 or AIS increased in the HPV arm in both age groups, the absolute difference in cumulative rates in women aged 35 years or older was small. By carefully selecting age groups and screening intervals, HPV screening could increase the overall detection rate of cervical precancerous lesions only slightly. However, these findings should be interpreted in the context of the high level of opportunistic screening that occurs in Finland.

Trial registration International Standard Randomised Controlled Trial ISRCTN23885553.

\section{Introduction}

Cervical cancer can be prevented by regular screening, but remains an important public health problem in Europe and beyond. ${ }^{12}$ The disease also affects young fertile women; thus, the effects on individual people and society can be greater than the effects of other cancers. It has also been claimed that current screening for cervical cancer has little or no effect on rates of invasive cancer at younger ages. ${ }^{3}$ The balance between positive health outcomes and adverse side effects of screening must be evaluated when considering new methods. These factors can be assessed best in the context of the overall burden of the disease in the target population.

DNA testing for human papillomavirus (HPV) has been shown to detect more preinvasive cervical lesions than standard cytology screening. ${ }^{4} \mathrm{HPV}$ screening can detect progressive 
lesions earlier, but also brings a risk of increased detection of non-progressive lesions. We assessed the detection rate of cervical lesions (both precancerous and cancerous), and their age specific patterns in two randomised cohorts. One cohort was screened initially by primary HPV DNA testing, and the other screened initially by conventional cytology (Pap test). We followed these cohorts over one screening round of five years.

\section{Methods}

\section{Randomisation and masking}

The target population was women invited for cervical cancer screening between 2003 and 2007 in southern Finland. Eligible women were drawn from the Population Information System (a national population register) by their birth year. Participants were randomly allocated (1:1) using computer generated random numbers, and invited to undergo primary screening for HPV DNA tests (with cytology triage for those with positive DNA results) or to undergo conventional cytology screening. The study design, methods of randomisation and invitation, screening protocols, and approval of the study have been previously described in detail. ${ }^{56}$

\section{Screening protocol}

We used a commercial HPV DNA test (Hybrid Capture 2, Qiagen), and provided cytology triage with a conventional Pap smear to women with positive DNA results (that is, a ratio of relative light units $\geq 1$ ). Pap smears were not read for those women with negative test results, unless they reported abnormal bleeding at the screening visit.

Women in both study arms were invited for screening using a similar letter with general information on screening. Using written material provided in the letter or at screening laboratories, women were informed of a new screening test in use that was at least as good as the Pap test. At the screening visit, the assigned test method was disclosed and the woman could decline the HPV test.

Cytology was reported using the Papanicolaou classification system in 2003-05 and the Bethesda system thereafter. Women were immediately referred for colposcopy if they had Papanicolaou classes III to V or squamous intraepithelial lesions of low to more severe grades. Papanicolaou class II or atypical squamous cells of undetermined significance were interpreted as borderline and indicated the need for intensive screening - that is, active surveillance with rescreening after 12-24 months. Intensive screening was also recommended for women who had positive results at HPV DNA testing (that is, were HPV positive) but had normal cytology.

Test results were posted to all participants within one month. Women in both arms received similar letters if their results were normal. For women recommended to intensive screening, the result was given in the letter; women recommended to referral for colposcopy were informed by phone. Nevertheless, the trial was conducted within the Finnish healthcare system, which has a distinguishing feature of large opportunistic screening. Women who participated in the programme had about the same number of opportunistic Pap smears (in the public or private healthcare service) as screening Pap tests. ${ }^{7}$

\section{Follow-up}

The exact date of invitation was not available in the screening register. Therefore, we used 1 January of each calendar year to approximate the time of randomisation, which closely related to the start of invitations. However, in reality, the randomisation had been done one to two months before this date. Thus, our final data did not include women who had been randomised but died, emigrated, or were diagnosed with invasive cervical cancer before invitation. The follow-up began from the approximated time of randomisation (that is, 1 January of each calendar year) and ended at the earliest date of: diagnosis of a cervical lesion, death, emigration, five years after randomisation, or 31 December 2008.

\section{Linkage procedure}

To obtain full information on the disease burden (that is, lesions found at screening and during the interval), we linked women individually between four registries using their personal identifiers. We obtained data for screening invitations, screening visits (including test results), and colposcopy referrals (with clinical or histological diagnoses done within the organised programme) from the Mass Screening Registry. The vital statistics and information on emigration was obtained from the Population Register Centre. The diagnosis of invasive cervical cancer was based on data from the Finnish Cancer Registry, which is a virtually complete database with regards to invasive disease. ${ }^{8}$ We also obtained information on precancerous lesions from the Care Registers for Social Welfare and Health Care (formerly the Finnish Hospital Discharge Register (HDR)).

We first obtained data from the Finnish Cancer Registry for the diagnosis of cervical intraepithelial neoplasia, grade 3 (CIN 3). CIN 3 is equal to dysplasia gravis and carcinoma in situ, and includes adenocarcinoma in situ (AIS) and epithelial neoplasm (not otherwise specified) in situ. We retrieved additional information from the Mass Screening Registry or HDR in case either database indicated the diagnoses of interest.

Data for CIN grade 1 lesions (CIN 1; equal to mild dysplasia) and grade 2 lesions (CIN 2; equal to moderate dysplasia) were available from the Mass Screening Registry or HDR only. We recorded only one precancerous lesion per woman. From the cervical lesions detected during follow-up, we chose the most severe lesion. If two or more registers indicated a cervical lesion of same stage, the earliest diagnosis was used. The date of diagnosis was taken directly from the Finnish Cancer Registry (for CIN 3, AIS, or invasive cervical cancer).

We obtained information on the earliest date of admission (including a diagnosis of cervical lesion of any malignancy grade) from the HDR. As a proxy for the date of diagnosis, we took information on the date of the screening visit from the Mass Screening Registry. Coverage of the screening register for detecting CIN lesions of grade 2 or higher severity (CIN $2+$ ) has been reported to be $99 \%$; corresponding coverage of the HDR has been reported to be $93 \% .^{8}$

CIN lesions in Finland are almost always histologically confirmed. During the three first years of the study, all cervical lesions of any grade (CIN 1+) were treated. From year 2006 onwards, Finnish guidelines recommended that CIN 2+ lesions be treated immediately, but that women younger than 30 years with CIN 1 lesions should be managed with surveillance until regression or treated if progression. However, clinical practices could vary regardless of the recommendation.

\section{Statistical analysis}

We used Poisson regression to assess the hazard (or detection) rate of cervical lesions by HPV screening, using cytology screening as the reference. We reported hazard ratio estimates with $95 \%$ confidence intervals stratified by index screen status and by age ( $<35$ years and $\geq 35$ years). The association between the detection rate of the cervical lesion and age, and the potential 
effect modification between age and screening method were tested using likelihood ratio statistics. Because the follow-up did not reach five years for most of the women, we analysed the five year cumulative hazard (that is, the cumulative detection rate) for precancerous and cancerous cervical lesions in both screening arms using the Nelson-Aalen estimator. Statistical analyses were based on random allocation for valid invitations throughout. All the statistical analyses were carried out using Stata (version 11.2, StataCorp).

This randomised trial on public health policy is registered as an International Standard Randomised Controlled Trial (ISRCTN23885553). Written informed consent from women was not required because the trial was conducted within the routine programme involving a large number of women.

\section{Results}

Of the 203788 women who were eligible for randomisation, $363(0.2 \%)$ were excluded because the end date of their follow-up was before 1 January of each screening year (fig $1 \Downarrow$ ). We invited 101678 women in the HPV screening arm and 101 747 in the conventional screening arm. Of the invited women, $66410(65.3 \%)$ in the HPV arm and $65785(64.7 \%)$ in the conventional arm attended screening. During an average observation period of 3.6 years (standard deviation 1.2, range $0-5)$, there were 724891 person years at risk for invasive cervical cancer and 720937 person years at risk for a cervical precancerous lesion. Table $1 \Downarrow$ presents the person years at risk (that is, person time), by study arm, attendance, and index screening status.

We recorded an equal number of referrals for colposcopy at the index screen in the HPV $(1.2 \%)$ and conventional (1.1\%) arms, but significantly more women were recommended for intensive screening after primary HPV DNA testing $(6.7 \%)$ than after primary cytology (5.7\%; table 1$)$. Slightly fewer women had negative results at index screen in the HPV arm than in the conventional arm $(92.1 \% \vee 93.1 \%)$.

During follow-up, in the HPV screening arm, we recorded 1010 cases of CIN 1+ lesions among women invited for screening and 766 among those attending. Corresponding numbers in the conventional screening arm were 701 and 446, respectively. Figure 1 and tables $2 \Downarrow$ and $3 \Downarrow$ show the number of cervical lesions by study arm, age, attendance, and index screening status.

Compared with the conventional screening arm, the hazard ratio of CIN 3 or AIS in the HPV screening arm was 1.32 (95\% confidence interval 1.09 to 1.59 ) among all invited women and 1.62 (1.28 to 2.06) among those who attended (table 2). More CIN 1 and CIN 2 lesions were detected by primary HPV testing than by primary cytology in their respective subgroups. The detection rate of invasive cervical cancer was also non-significantly increased in women who underwent HPV screening, compared with conventional screening (table 2). Among women who did not attend screening, fewer cases of invasive cervical cancer were diagnosed in the HPV arm than in the cytology arm during follow-up $(0.37$ (95\% confidence interval 0.17 to 0.83 ), table 2 ), whereas no difference was seen between screening arms for less severe cervical outcomes (CIN $1+$ or AIS)

More cervical precancerous lesions were detected by HPV testing than by cytology in women who were referred directly to colposcopy and those who were recommended for intensive screening (table 3). The detection rate of CIN 3 or AIS was significantly lower in women who had a negative result after HPV screening than in women with normal cytology (hazard ratio 0.32 (95\% confidence interval 0.13 to 0.79$)$ ). The decreased detection of CIN 2 ( 0.65 ( 0.37 to 1.13$)$ ) was not significant, and no decrease was observed for CIN 1 (1.18 (0.67 to 2.09); table 3).

For women aged 25-34 years, the cumulative hazard of CIN 3 or AIS during the five year period was 0.0057 (95\% confidence interval 0.0045 to 0.0072 ) in the HPV screening arm and 0.0046 ( 0.0035 to 0.0059$)$ in the conventional screening arm. For women aged $\geq 35$ years, the cumulative hazards were 0.0022 (0.0019 to 0.0026$)$ and 0.0017 (0.0014 to 0.0021$)$, respectively (fig $2 \Downarrow$ ). The cumulative hazards of CIN 1 and CIN 2 were higher in the younger age group than in the older group, in both screening arms (table $4 \Downarrow$ ). In women aged 35 years or older, there was only a small absolute increase in the cumulative hazard of mild and moderate precancerous lesions in the HPV arm, compared with the cytology arm (table 4 , figs $3 \Downarrow$ and $4 \Downarrow$ ).

\section{Discussion}

Overall, primary screening for HPV DNA detected more cervical cancer lesions than primary cytology within one screening round of five year. Primary HPV screening resulted in the diagnosis of more CIN lesions than conventional cytology in women referred directly to colposcopy and in those recommended to intensive screening. Compared with older women, both screening arms had a substantial excess of CIN lesions in those aged 25-34 years. Detection of CIN 3 or AIS was strongly decreased among women who had a negative result from the HPV DNA test, compared with women who had a normal Pap test result, in all ages.

In our study, we did not have consistent information on the effect of HPV screening on cervical cancer incidence, owing to the short follow-up. The difference in detection rates of CIN or invasive cervical cancer between the two screening methods was largest at three years after randomisation to screening. This difference was probably due to programme initiated testing, which leads to extra tests within one to two years after the index screen (that is, at 2-3 years from the start of follow-up). Among women aged 35 years or older, very few cases of CIN 3 or AIS were diagnosed later than three and half years after invitation in the HPV arm (fig 4). By contrast, we saw a constant increase in the detection of CIN 3 or AIS in the conventional screening arm between two and five years of follow-up. This difference in rates could indicate a diagnosis of high grade cervical lesions with the HPV DNA test earlier than was thought previously. ${ }^{9-11}$ At the same time, the absolute difference in the cumulative rate of CIN 3 or AIS detection in women aged 35 years or older increased only slightly within the five year screening interval. These findings suggest that by carefully selecting age groups and screening intervals, HPV screening may increase the overall detection rate of cervical precancerous lesions only slightly.

\section{Strengths and limitations}

This Finnish trial is one of the largest HPV screening trials and was specifically designed to assess effectiveness in the national service screening programme. The attendance rate in the study area was $65 \%$, which is typical for southern Finland. However, the study had a few limitations.

The trial was conducted within the Finnish healthcare system with large numbers of opportunistic Pap testing. The results reported here reflect test performance within this context; therefore extrapolations to other environments should be done with caution. In the HDR, every contact to the healthcare service is recorded with some diagnosis. Therefore, it was impossible to distinguish incident cervical lesions from prevalent ones. ${ }^{8}$ 
From the HDR, we chose the most severe diagnosis and accompanied it with the earliest date of admission that included cervical lesion of any grade. Also, we tried to minimise over-reporting by excluding the cervical lesions that were prevalent at the start of the study. It is likely that some over-reporting still remained, but owing to randomisation, over-reporting should have been of the same size in both screening arms.

Cervical cancer incidence was substantially lower in the HPV arm than in the conventional arm among women who did not attend screening. According to the protocol, the screening method was disclosed and explained at the screening visit. However, some women could have received information about the method before their decision to participate, which could have lead to selection in attendance. Also, it was not possible to know what kind of information the women had obtained themselves about the screening tests beforehand, and whether this had influenced their decision to accept the HPV test or request the Pap test instead.

Another explanation could be that randomisation had failed. We made efforts to identify any technical error in the randomising process itself (including independent checking of the randomisation procedure, accumulation of women in the study arms during follow-up, and checking of previous screening history), but did not found any. Moreover, an error in randomisation should also show in the detection rates and screening history of women before the study period; no such difference was observed (data not shown). Therefore, we cannot rule out pure chance even though the difference was statistically significant.

Lastly, in the current study, we assessed cumulative disease rates. In addition to screen detected cases, we based these rates on a mixture of intensive testing (from programme initiated surveillance) and opportunistic testing (conducted outside the programme). Opportunistic Pap testing would preferentially improve the Pap test performance because the primary screening test with the higher detection rate (the HPV DNA test) would identify more disease, which would be treated and removed from the study arm, thus leading to a different occurrence of preinvasive lesions between the arms. During follow-up, this effect favours disease detection in the screening method with the higher residual incidence; the bias is not controlled by randomisation because the different prevalences of disease were created afterwards. However, since the HPV screening arm still had a higher prevalence than the conventional screening arm, this bias does not explain our result concerning the cumulative rate at five years. In fact, the difference between study arms did not increase after three years of follow-up.

\section{Comparison with previous studies}

For women with negative results, we estimated that the cumulative hazard of CIN 3 or AIS after HPV screening was about a third of the cumulative hazard after cytology screening, over five years (fig 2). This decrease was roughly the same for women aged 25-34 years at screening as well as those aged 35-65 years. The joint European cohort study reported a similar ratio for the cumulative incidence of CIN $3+$ (that is, CIN 3 and invasive cancer, including squamous cell carcinoma and adenocarcinoma) after five years between women who were negative for HPV and those who were negative for cytology $(0.25 \% v 0.83 \%){ }^{11}$

After comparing cervical precancerous lesions detected after a borderline test result at index screen, we saw a large difference between screening methods. Among women who were HPV positive but cytology negative, 58 cases of CIN $3+$ lesions were detected, compared with only 16 cases after borderline cytology in the conventional arm. Furthermore, the detection rate of CIN 2 lesions among women who were initially HPV positive but cytology negative was much higher than the detection rate after negative or borderline cytology in the conventional arm. This difference was due to the study protocol, because HPV positive women were rescreened after one to two years and those who were persistently HPV positive were referred to colposcopy after two or three consecutive positive results, even if cytology was normal. Furthermore, women with borderline cytology in the conventional arm were rescreened, and two or three consecutive borderline results triggered a referral for colposcopy..$^{5}$

The HPV screening arm had slightly more incident cases of cervical cancer diagnosed at the index screen and rescreening visits, compared with the conventional screening arm. During follow-up, no cases of invasive cervical cancer were diagnosed in the conventional screening arm after borderline cytology and two cases of invasive cervical cancer were diagnosed after negative cytology (one squamous cell carcinoma and one adenocarcinoma). In the HPV screening arm, three cases of invasive cervical cancer were diagnosed among HPV positive women who had normal or borderline cytology at the baseline and five cancers after a negative HPV test. Of these, six were adenocarcinomas and two of squamous origin. Screening with cytology has been shown to be less effective in preventing adenocarcinomas than those of squamous origin. ${ }^{12}{ }^{13}$ This finding might also be true with HPV screening followed by cytology triage.

European guidelines for quality assurance in cervical cancer screening ranked indicators of screening effectiveness using a six tier scale. ${ }^{14}$ Thus far, the highest level of evidence- that is, a decrease in deaths from cervical cancer-has been reported from India after eight years of follow-up. ${ }^{15}$ Lynge and colleagues wrote that a switch to HPV testing in primary screening in Europe is likely if the screening trials show reduction in detection rates of $\mathrm{CIN} 3+$ at the subsequent screening round and if HPV testing does not increase the burden of follow-up and treatment for participating women. ${ }^{16}$ Several studies from Europe and North America have already shown a reduced incidence of CIN 3+. ${ }^{9-11} 1718$ Thus far, two European trials have reported decreased risk of cervical cancer in HPV screening compared with cytology screening. ${ }^{17} 19$ These results also include cancers diagnosed at the second round of screening. Owing to gradual implementation and a screening interval of five years only, our data do not include results from the second round.

The POBASCAM trial reported no difference in the cumulative detection of CIN 3+ and CIN 2+ between younger (aged 29-33 years) and older women during two screening rounds. ${ }^{19}$ This trial integrated primary HPV testing into the screening programme in a different manner to our study; in the second screening round, women were screened by primary HPV testing in both arms. Our data showed that the cumulative hazard of cervical precancerous lesions was substantially higher in women aged 25-34 years than in women aged 35 years or older, especially in the HPV screening arm. Although the Hybrid Capture 2 test used in our study and the GP5+/6+ polymerase chain reaction method used in the POBASCAM study are both clinically validated and have similar performances, the slight difference in clinical specificity between the two tests might partly explain the difference. ${ }^{20}$ Additionally, our intensive procedures for repeat testing after a positive HPV test could have a role, and we should assess the optimal management procedure in the future. 


\section{Conclusions and policy implications}

Owing to the use of different registries and delays in registration, it was not possible to assess whether cervical lesions were diagnosed during intensive screening of the programme or diagnosed outside of the programme but initiated by the entry screening test result. Also, many women with normal test results were screened outside the programme between two screening rounds. Currently, only a third of the annual diagnoses of any CIN lesions in Finland are detected by the national programme. Furthermore, opportunistic screening is more expensive, whereas detecting lesions using an organised programme would be more cost effective. ${ }^{7}$ Thus, when introducing primary HPV screening, it would be essential to reduce opportunistic screening, especially for young women in whom mild cervical lesions and spontaneous regression are common. ${ }^{21}$ Currently in Finland, CIN 1 lesions are treated if they persist for 24 months but CIN 2 or more severe lesions are usually immediately treated.

However, even two thirds of CIN 3 lesions will not progress to invasive cancer within 30 years. ${ }^{22}$ In cervical cancer screening, triage use of Pap smears after a positive HPV result has been seen as the best screening strategy in countries that undertake cytology of good quality. ${ }^{23}$ However, new management strategies should also be studied, particularly if considering a switch to primary HPV testing. For example, surveillance could be a more appropriate approach for CIN 2 lesions, specifically at young ages when progression of precancerous lesions is less common.

We thank Timo Hakulinen and Tapio Luostarinen from the Finnish Cancer Registry for checking randomisation procedures in relation to the attendance status of the current study; Timo Hakulinen for his help with the statistical analysis; and Qiagen for providing the Hybrid Capture 2 tests at a long term developmental price, and checking the technical details of their product for this manuscript.

Contributors: MKL planned the design and method, monitored data collection and management, analysed the data, and drafted and revised the paper. PN planned the design and method, monitored laboratory analyses, and drafted and revised the paper. SL monitored data management and revised the paper. $\mathrm{NM}$ and $\mathrm{MH}$ monitored data validity and revised the paper. AP wrote the statistical analysis plan, analysed the data, and revised the paper. PL monitored laboratory analyses and revised the paper. JT monitored laboratory analyses and revised the paper. AA planned the design and method, monitored data collection and management, analysed the data, and drafted and revised the paper. MKL and AA are guarantors. MKL had full access to all the data in the study and decided to submit the manuscript for publication.

Funding: This study was supported by research grants from the Academy of Finland, the European Union through the action programme Europe Against Cancer, the Finnish Cancer Organisations, and the Finnish Medical Society Duodecim. The funders had no role in study design, data collection, data analysis, data interpretation, writing of the report or the decision to submit it for publication.

Competing interests: All authors have completed the ICMJE uniform disclosure form at www.icmje.org/coi_disclosure.pdf (available on request from the corresponding author) and declare: support from the Academy of Finland, the European Union through the action programme Europe Against Cancer, and the Finnish Cancer Organisations, and the Finnish Medical Society Duodecim for the submitted work; PN has done consultancy work for GlaxoSmithKline and Merck; and no other relationships or activities that could appear to have influenced the submitted work.
Ethical approval: This study was approved by principal authorities in medical and ethical issues in Finland (for example, the National Authority for Medicolegal Affairs (Dnro 3950/32/300/02; Dnro

THL/1223/6.02.00/2010), the ethical committee of the local hospital district (Dnro 221/E8/02; 384/13/03/032010), and health boards of the committed municipalities.

Data sharing: No additional data available.

1 International Agency for Research on Cancer. Human papillomaviruses. IARC Monographs on the evaluation of carcinogenic risks to humans. IARC Press, 2007.

2 Arbyn M, Raifu AO, Weiderpass E, Bray F, Anttila A. Trends of cervical cancer mortality in the member states of the European Union. Eur J Cancer 2009;45:2640-8.

3 Sasieni P, Castanon A, Cuzick J. Effectiveness of cervical screening with age: population based case-control study of prospectively recorded data [correction in: $B M J$ 2009;339:b3115]. BMJ 2009;339:b2968.

4 Cuzick J, Arbyn M, Sankaranarayanan R, Tsu V, Ronco G, Mayrand MH, et al. Overview of human papillomavirus-based and other novel options for cervical cancer screening in developed and developing countries. Vaccine 2008;26(suppl 10):K29-41.

5 Leinonen M, Nieminen P, Kotaniemi-Talonen L, Malila N, Tarkkanen J, Laurila P, et al. Age-specific evaluation of primary human papillomavirus screening vs conventional cytology in a randomised setting. J Natl Cancer Inst 2009;101:1612-23.

6 Anttila A, Hakama M, Kotaniemi-Talonen L, Nieminen P. Alternative technologies in cervical cancer screening: a randomized evaluation trial. BMC Public Health 2006;16:252.

7 Working group of human papillomavirus diseases. The National Institute for Health and Welfare. Report 28/2011. www.thl.fi/thl-client/pdfs/94d6f45d-22e1-4b53-b6152eea48d90e1c.

8 Lönnberg S, Leinonen M, Malila N, Anttila A. Validation of histological diagnoses in a national cervical screening register. Acta Oncol 2012;51:37-44.

9 Naucler $\mathrm{P}$, Ryd W, Törnberg S, Strand A, Wadell G, Elfgren K, et al. Human papillomavirus and Papanicolaou tests to screen for cervical cancer. N Engl J Med 2007;357:1589-97.

10 Bulkmans NW, Berkhof J, Rozendaal L, van Kemenade FJ, Boeke AJ, Bulk S, et al. Human papillomavirus DNA testing for the detection of cervical intraepithelial neoplasia grade 3 and cancer: 5-year follow-up of a randomized controlled implementation trial. Lancet 2007;370:1764-72.

11 Dillner J, Rebolj M, Birembaut P, Petry KU, Szarewski A, Munk C, et al. Long term predictive values of cytology and human papillomavirus testing in cervical cancer screening: joint European cohort study. BMJ 2008;377:a1754.

12 Nieminen $P$, Kallio M, Hakama M. The effect of mass screening on incidence and mortality of squamous and adenocarcinoma of cervix uteri. Obstet Gynecol 1995;85:1017-21.

13 Sasieni P, Castanon A, Cuzick J. Screening and adenocarcinoma of the cervix. Int J Cancer 2009;125:525-9.

14 Arbyn M, Anttila A, Jordan J, Ronco G, Schenck U, Segnan N, Wiener HG, Herbert A Daniel J, von Karsa L. European guidelines for quality assurance in cervical cancer screening. Office for official publications of the European Communities, 2008.

15 Sankaranarayanan R, Nene BM, Shastri SS, Jayant K, Muwonge R, Budukh AM, et al. HPV screening for cervical cancer in rural India. N Engl J Med 2009;360:1385-94.

16 Lynge E, Anttila A, Arbyn M, Segnan N, Ronco G. What's next? Perspectives and future needs of cervical screening in Europe in the era of molecular testing and vaccination. Eur $J$ Cancer 2009;45:2714-21.

17 Ronco G, Giorgi-Rossi P, Carozzi F, Confortini M, Dalla Palma P, Del Mistro A, et al. Efficacy of human papillomavirus testing for the detection of invasive cervical cancers and cervical intraepithelial neoplasia: a randomised controlled trial. Lancet Oncol 2010;11:249-57.

18 Kitchener HC, Almonte M, Thomson C, Wheeler P, Sargent A, Stoykova B, et al. HPV testing in combination with liquid-based cytology in primary cervical screening (ARTISTIC): a randomised controlled trial [correction in: Lancet Oncol 2009;10:748]. Lancet Oncol 2009;10:672-82.

19 Rijkaart DC, Berkhof J, Rozendaal L, van Kemenade FJ, Bulkmans NW, Heideman DA, et al. Human papillomavirus testing for the detection of high-grade cervical intraepithelial neoplasia and cancer: final results of the POBASCAM randomised controlled trial. Lancet Oncol 2012;13:78-88.

20 Meijer CJ, Berkhof J, Castle PE, Hesselink AT, Franco EL, Ronco G, et al. Guidelines for human papillomavirus DNA test requirements for primary cervical cancer screening in women 30 years and older. Int $J$ Cancer 2009;124:516-20.

21 Moscicki AB, Shiboski S, Hills NK, Powell KJ, Jay N, Hanson EN, et al. Regression of low-grade squamous intraepithelial lesions in young women. Lancet 2004;364:1678-83.

22 McCredie MR, Sharples KJ, Paul C, Baranyai J, Medley G, Jones RW, et al. Natural history of cervical neoplasia and risk of invasive cancer in women with cervical intraepithelial neoplasia 3: a retrospective cohort study. Lancet Oncol 2008;9:425-34.

23 Naucler P, Ryd W, Törnberg S, Strand A, Wadell G, Elfgren K, et al. Efficacy of HPV DNA testing with cytology triage and/or repeat HPV DNA testing in primary cervical cancer screening. J Natl Cancer Inst 2009;101:88-99.

Accepted: 08 November 2012

\section{Cite this as: BMJ 2012;345:e7789}

This is an open-access article distributed under the terms of the Creative Commons Attribution Non-commercial License, which permits use, distribution, and reproduction in any medium, provided the original work is properly cited, the use is non commercial and is otherwise in compliance with the license. See: http://creativecommons.org/licenses/bync/2.0/ and http://creativecommons.org/licenses/by-nc/2.0/legalcode. 


\section{What is already known on this topic}

Human papillomavirus (HPV) DNA testing is more sensitive and detects progressive cervical lesions earlier than conventional cytology, but also brings a risk of increased detection of non-progressive lesions

A positive result from HPV tests does not necessarily indicate an immediate need for confirmation and treatment, because most infections will regress rapidly without causing significant cellular atypia

Triage use of Pap smears after a positive HPV result has been seen as the appropriate screening strategy in countries where cytology is of good quality

\section{What this study adds}

The cumulative detection rate of cervical intraepithelial neoplasia (CIN) lesions increased only slightly in women aged 35 years or more More CIN lesions were detected in women younger than 35 years and were substantially more common by HPV test than by cytology screening

By carefully selecting age groups and screening intervals, HPV screening may increase the overall detection rate of cervical precancerous lesions only slightly

\section{Tables}

\begin{tabular}{|c|c|c|c|c|c|c|}
\hline \multirow[b]{3}{*}{$\begin{array}{l}\text { Participant status, by age } \\
\text { group at randomisation }\end{array}$} & \multicolumn{3}{|c|}{ HPV screening arm } & \multicolumn{3}{|c|}{ Conventional screening arm } \\
\hline & \multirow[b]{2}{*}{ No of women } & \multicolumn{2}{|c|}{ Person years at risk (\%) } & \multirow[b]{2}{*}{ No of women } & \multicolumn{2}{|c|}{ Person years at risk (\%) } \\
\hline & & $\begin{array}{l}\text { Invasive cervical } \\
\text { cancer }\end{array}$ & $\begin{array}{l}\text { Cervical lesion of any } \\
\text { grade }^{*}\end{array}$ & & $\begin{array}{l}\text { Invasive cervical } \\
\text { cancer }\end{array}$ & $\begin{array}{c}\text { Cervical lesion of any } \\
\text { grade* }^{\star}\end{array}$ \\
\hline $\begin{array}{l}\text { Valid invitations to attend } \\
\text { screening }\end{array}$ & 101678 & $362318(100.0)$ & $360017(100.0)$ & 101747 & $362573(100.0)$ & $360919(100.0)$ \\
\hline 25-34 years & 20460 & 71512 & 70553 & 20455 & 71548 & 70882 \\
\hline $35-65$ years & 81218 & 290806 & 289464 & 81292 & 291025 & 290037 \\
\hline Women who attended & 66410 & $238714(65.9)$ & $236946(65.8)$ & 65784 & $236548(65.2)$ & 235431 (65.2) \\
\hline 25-34 years & 11191 & 39620 & 38913 & 11071 & 38942 & 38535 \\
\hline $35-65$ years & 55219 & 199094 & 198033 & 54713 & 197606 & 196896 \\
\hline Women who did not attend & 35268 & $123603(34.1)$ & $123071(34.2)$ & 35963 & $126025(34.8)$ & $125488(34.8)$ \\
\hline $25-34$ years & 9269 & 31891 & 31641 & 9384 & 32606 & 32347 \\
\hline $35-65$ years & 25999 & 91712 & 91430 & 26579 & 93419 & 93141 \\
\hline $\begin{array}{l}\text { Colposcopy referral at index } \\
\text { screen }\end{array}$ & 796 & $2842(0.8)$ & $1559(0.4)$ & 755 & $2670(0.7)$ & $1787(0.5)$ \\
\hline 25-34 years & 290 & 1066 & 548 & 211 & 767 & 443 \\
\hline $35-65$ years & 506 & 1776 & 1011 & 544 & 1903 & 1344 \\
\hline $\begin{array}{l}\text { Intensive screening at index } \\
\text { screen }\end{array}$ & 4449 & 15909 (4.4) & $15510(4.3)$ & 3755 & 13515 (3.7) & 13400 (3.7) \\
\hline 25-34 years & 1686 & 5889 & 5724 & 669 & 2323 & 2297 \\
\hline $35-65$ years & 2763 & 10020 & 9787 & 3086 & 11192 & 11103 \\
\hline $\begin{array}{l}\text { Negative or normal findings } \\
\text { at index screen }\end{array}$ & 61165 & $219963(60.7)$ & $219876(61.1)$ & 61274 & $220362(60.8)$ & $220244(61.0)$ \\
\hline 25-34 years & 9215 & 32665 & 32641 & 10191 & 35852 & 35795 \\
\hline $35-65$ years & 51950 & 187298 & 187236 & 51083 & 184510 & 184449 \\
\hline
\end{tabular}


Table 2| Hazard ratios of invasive cervical cancer (ICC), AIS, or CIN among women invited for organised cervical screening in Finland, by screening status

\begin{tabular}{|c|c|c|c|c|c|c|c|}
\hline & \multicolumn{2}{|c|}{$\begin{array}{c}\text { No of cases detected in } \\
\text { women } \\
\text { aged 25-34 years }\end{array}$} & \multicolumn{2}{|c|}{$\begin{array}{c}\text { No of cases detected in } \\
\text { women } \\
\text { aged } \geq 35 \text { years }\end{array}$} & \multicolumn{3}{|c|}{ Hazard ratio $(95 \% \mathrm{Cl})$} \\
\hline & $\begin{array}{c}\text { HPV } \\
\text { screening } \\
\text { arm }\end{array}$ & $\begin{array}{l}\text { Conventional } \\
\text { screening arm }\end{array}$ & $\begin{array}{c}\text { HPV } \\
\text { screening arm }\end{array}$ & $\begin{array}{l}\text { Conventional } \\
\text { screening arm }\end{array}$ & Age $25-34$ years & Age $\geq 35$ years & Overall \\
\hline \multicolumn{8}{|c|}{ All women invited } \\
\hline ICC & 1 & 6 & 24 & 25 & 0.17 (0.02 to 1.39 ) & 0.96 (0.55 to 1.68$)$ & 0.81 (0.48 to 1.37 ) \\
\hline CIN 3 or AIS & 90 & 70 & 158 & 119 & 1.29 (0.95 to 1.77$)$ & $1.33(1.05$ to 1.69$)$ & 1.32 (1.09 to 1.59$)$ \\
\hline CIN 2 & 209 & 135 & 236 & 155 & 1.56 (1.25 to 1.93$)$ & $1.53(1.25$ to 1.87$)$ & 1.54 (1.33 to 1.78$)$ \\
\hline CIN 1 & 117 & 68 & 175 & 123 & 1.73 (1.28 to 2.33$)$ & $1.43(1.13$ to 1.80$)$ & 1.53 (1.28 to 1.84$)$ \\
\hline \multicolumn{8}{|c|}{ Women who attended } \\
\hline ICC & 1 & 2 & 16 & 7 & 0.49 (0.04 to 5.42$)$ & 2.27 (0.93 to 5.51$)$ & 1.87 (0.83 to 4.20$)$ \\
\hline CIN 3 or AIS & 62 & 32 & 116 & 77 & $1.92(1.25$ to 2.94$)$ & $1.50(1.12$ to 2.00$)$ & 1.62 (1.28 to 2.06$)$ \\
\hline CIN 2 & 155 & 85 & 190 & 116 & 1.81 (1.39 to 2.35$)$ & $1.63(1.29$ to 2.05$)$ & 1.71 (1.43 to 2.03$)$ \\
\hline CIN 1 & 88 & 43 & 138 & 84 & 2.03 (1.41 to 2.92$)$ & 1.63 (1.25 to 2.14$)$ & 1.77 (1.42 to 2.20$)$ \\
\hline \multicolumn{8}{|c|}{ Women who did not attend } \\
\hline ICC & 0 & 4 & 8 & 18 & NA & 0.45 (0.20 to 1.04$)$ & $0.37(0.17$ to 0.83$)$ \\
\hline CIN 3 or AIS & 28 & 38 & 42 & 42 & 0.75 (0.46 to 1.23$)$ & $1.02(0.66$ to 1.56$)$ & $0 \square 89(0.65$ to 1.23$)$ \\
\hline $\mathrm{CIN} 2$ & 54 & 50 & 46 & 39 & 1.10 (0.75 to 1.62$)$ & $1.20(0.78$ to 1.84$)$ & 1.15 (0.86 to 1.52$)$ \\
\hline CIN 1 & 29 & 25 & 37 & 39 & 1.19 (0.69 to 2.02 ) & 0.97 (0.62 to 1.52$)$ & 1.05 (0.75 to 1.48$)$ \\
\hline
\end{tabular}

$\mathrm{NA}=$ not applicable. Association between age and ICC was not significant $(\mathrm{P}=0.83$ among all invited, $\mathrm{P}=0.96$ among women who attended, and $\mathrm{P}=0.12$ among women who did not attend). Associations between age and $\mathrm{CIN} 1, \mathrm{CIN} 2$, and $\mathrm{CIN} 3$ or AIS were significant ( $<<0.001$ among all subgroups of attendance). There was significant effect modification between age and screening method only for $\mathrm{CIN} 1$ among all women invited $(\mathrm{P}=0.002)$ and among women who attended $(\mathrm{P}=0.004)$. 
Table 3| Hazard ratios of invasive cervical cancer (ICC), AIS, or CIN among women who attended organised cervical screening in Finland, by status at index screen

\begin{tabular}{|c|c|c|c|c|c|c|c|}
\hline & \multicolumn{2}{|c|}{$\begin{array}{l}\text { No of cases detected in } \\
\text { women aged } 25-34 \text { years }\end{array}$} & \multicolumn{2}{|c|}{$\begin{array}{l}\text { No of cases detected in } \\
\text { women aged } \geq 35 \text { years }\end{array}$} & \multicolumn{3}{|c|}{ Hazard ratio $(95 \% \mathrm{Cl})$} \\
\hline & $\begin{array}{c}\text { HPV } \\
\text { screening } \\
\text { arm }\end{array}$ & $\begin{array}{l}\text { Conventional } \\
\text { screening arm }\end{array}$ & $\begin{array}{c}\text { HPV } \\
\text { screening } \\
\text { arm }\end{array}$ & $\begin{array}{l}\text { Conventional } \\
\text { screening arm }\end{array}$ & Age $25-34$ years & Age $\geq 35$ years & Overall \\
\hline \multicolumn{8}{|c|}{ Colposcopy referral } \\
\hline ICC & 1 & 1 & 8 & 6 & $0.72(0.05$ to 11.5$)$ & 1.43 (0.50 to 4.12$)$ & $1.21(0.45$ to 3.24$)$ \\
\hline CIN 3 or AIS & 37 & 20 & 80 & 54 & 1.50 (0.87 to 2.58$)$ & 1.97 (1.39 to 2.78$)$ & 1.81 (1.35 to 2.43 ) \\
\hline CIN 2 & 85 & 59 & 106 & 85 & 1.17 (0.84 to 1.62$)$ & $1.66(1.25$ to 2.20$)$ & $1.52(1.22$ to 1.89$)$ \\
\hline CIN 1 & 47 & 26 & 70 & 52 & 1.46 (0.91 to 2.36 ) & 1.79 (1.25 to 2.56$)$ & 1.72 (1.29 to 2.29$)$ \\
\hline \multicolumn{8}{|c|}{ Intensive screening } \\
\hline ICC & 0 & 0 & 3 & 0 & NA & NA & NA \\
\hline CIN 3 or AIS & 23 & 2 & 32 & 14 & 4.61 (1.09 to 19.6$)$ & 2.59 (1.38 to 4.86$)$ & 2.97 (1.70 to 5.18$)$ \\
\hline $\mathrm{CIN} 2$ & 64 & 11 & 70 & 15 & 2.33 (1.23 to 4.43$)$ & 5.29 (3.03 to 9.25$)$ & 4.45 (2.93 to 6.78$)$ \\
\hline CIN 1 & 34 & 6 & 49 & 21 & 2.27 (0.95 to 5.42$)$ & 2.65 (1.59 to 4.41$)$ & 2.66 (1.72 to 4.10$)$ \\
\hline \multicolumn{8}{|c|}{ Negative/normal findings } \\
\hline ICC & 0 & 1 & 5 & 1 & NA & 4.93 (0.58 to 42.2 ) & 2.50 (0.49 to 12.9$)$ \\
\hline CIN 3 or AIS & 2 & 10 & 4 & 9 & $0.22(0.05$ to 1.00$)$ & 0.44 (0.13 to 1.42$)$ & $0.32(0.13$ to 0.79$)$ \\
\hline CIN 2 & 6 & 15 & 14 & 16 & 0.44 (0.17 to 1.13$)$ & 0.86 (0.42 to 1.77$)$ & 0.65 (0.37 to 1.13$)$ \\
\hline CIN 1 & 7 & 11 & 19 & 11 & $0.70(0.27$ to 1.80$)$ & $1.70(0.81$ to 3.58$)$ & 1.18 (0.67 to 2.09$)$ \\
\hline
\end{tabular}

$\mathrm{NA}=$ not applicable. There was significant association between age and CIN 1, CIN 2, and CIN 3 or AIS among all statuses at index screen; association between age and ICC was significant only among women referred to colposcopy $(P=0.04)$. The effect modification between age and screening method was seen only for $\mathrm{CIN} 1$ among women referred for colposcopy $(\mathrm{P}=0.01)$ and those with negative or normal findings $(\mathrm{P}=0.04)$, but not among subgroup of intensive screening $(\mathrm{P}=0.11)$. 
Table 4| Cumulative hazard $(95 \% \mathrm{Cl}$ ) of CIN or AIS per 10000 person years among women invited for organised cervical screening in Finland

Age 25-34 years at randomisation

HPV screening arm Conventional screening arm

\begin{tabular}{lclll} 
CIN 3 or AIS & $57(45$ to 72$)$ & $46(35$ to 59$)$ & $22(19$ to 26$)$ & $17(14$ to 21$)$ \\
\hline CIN 2 & $120(110$ to 140$)$ & $80(66$ to 96$)$ & $37(32$ to 42$)$ & $23(19$ to 27$)$ \\
\hline CIN 1 & $74(60$ to 91$)$ & $44(34$ to 58$)$ & $27(23$ to 32$)$ & $19(16$ to 23$)$
\end{tabular}




\section{Figures}

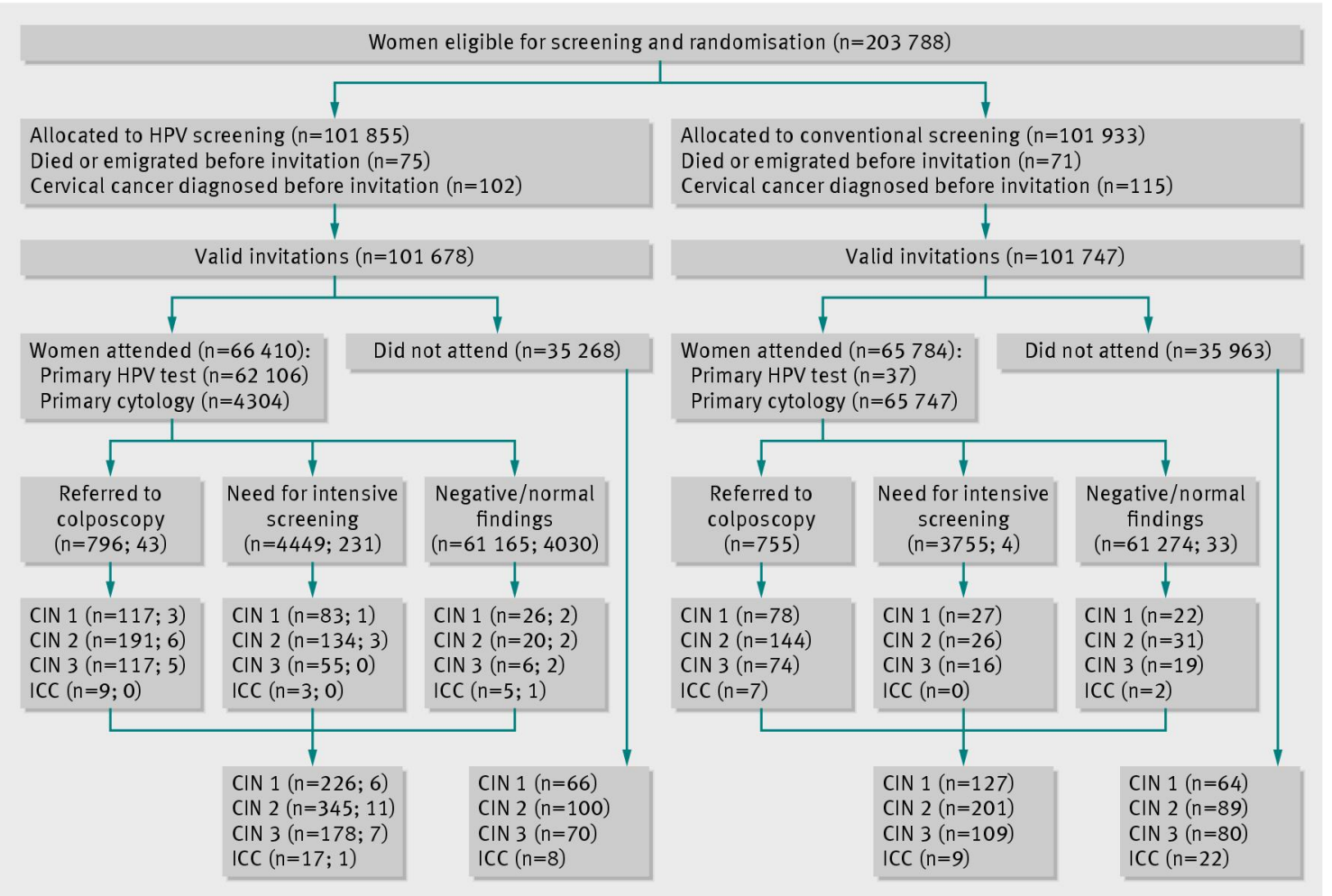

Fig 1 Flowchart of the screening profiles of women invited for cervical screening in the Finnish HPV screening trial in 2003-07. ICC=invasive cervical cancer. Data after semi colons are number of women who were not screened according to their random allocation

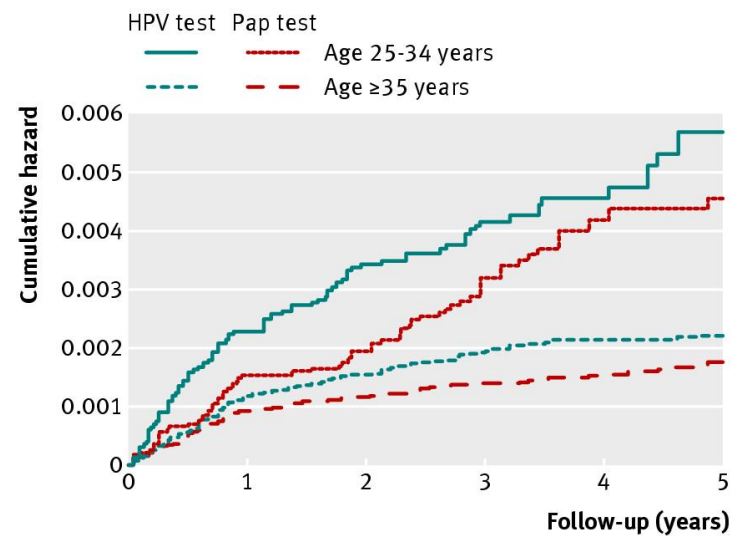

Fig 2 Cumulative hazard (or cumulative detection rate) of CIN 3 or AIS among women invited for cervical screening by age group at randomisation and by screening arm 


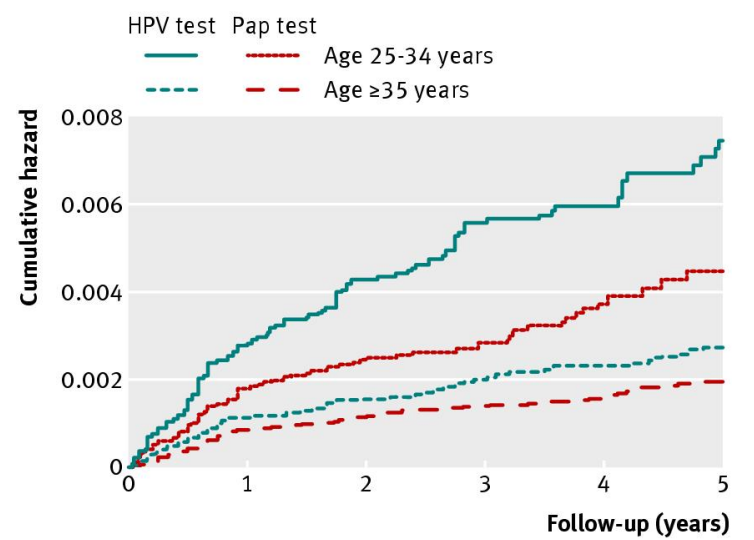

Fig 3 Cumulative hazard (or cumulative detection rate) of CIN 1 among women invited for cervical screening by age group at randomisation and by screening arm

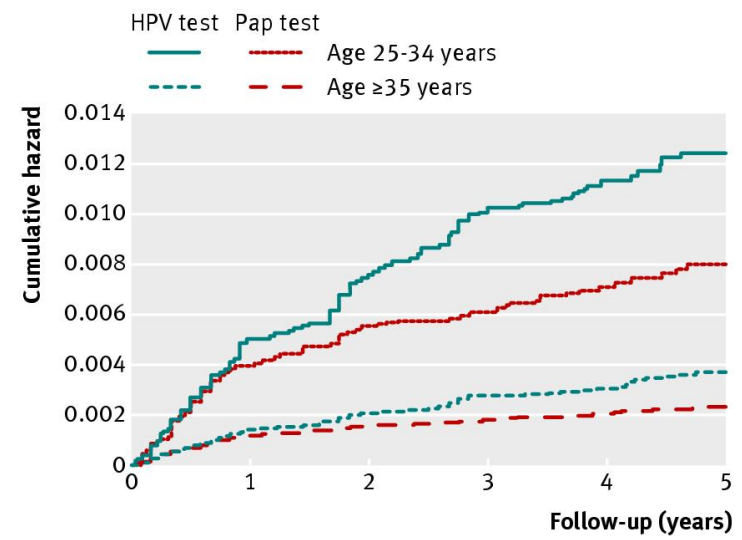

Fig 4 Cumulative hazard (or cumulative detection rate) of CIN 2 among women invited for cervical screening by age group at randomisation and by screening arm 